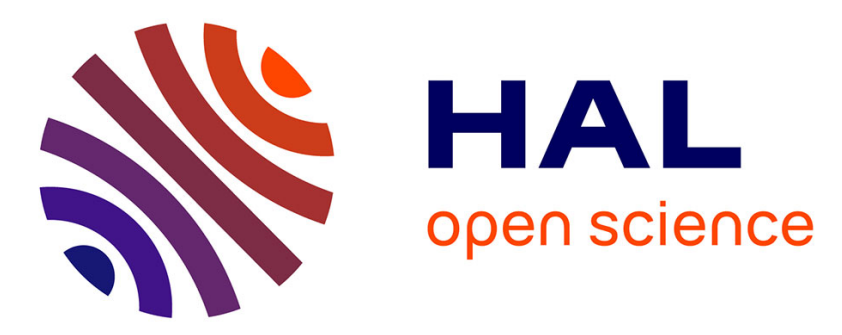

\title{
Résultat d'un entraînement en conscience phonologique chez des élèves en difficultés de lecture au cycle 3
}

Sophie Briquet-Duhazé, Amine Rezrazi

\section{To cite this version:}

Sophie Briquet-Duhazé, Amine Rezrazi. Résultat d'un entraînement en conscience phonologique chez des élèves en difficultés de lecture au cycle 3. Enfance, 2014, 2014 (02), pp.119-134. 10.3917/enf1.142.0119 . hal-01905514

\section{HAL Id: hal-01905514 \\ https://hal.science/hal-01905514}

Submitted on 25 Oct 2018

HAL is a multi-disciplinary open access archive for the deposit and dissemination of scientific research documents, whether they are published or not. The documents may come from teaching and research institutions in France or abroad, or from public or private research centers.
L'archive ouverte pluridisciplinaire HAL, est destinée au dépôt et à la diffusion de documents scientifiques de niveau recherche, publiés ou non, émanant des établissements d'enseignement et de recherche français ou étrangers, des laboratoires publics ou privés. 


\section{RÉSULTAT D'UN ENTRAÎNEMENT EN CONSCIENCE PHONOLOGIQUE CHEZ DES ÉLÈVES EN DIFFICULTÉS DE LECTURE AU CYCLE 3 \\ Sophie Briquet-Duhazé, Amine Rezrazi}

NecPlus | « Enfance »

2014/2 N² | pages 119 à 134

ISSN 0013-7545

Article disponible en ligne à l'adresse :

https://www.cairn.info/revue-enfance2-2014-2-page-119.htm

\section{Pour citer cet article :}

Sophie Briquet-Duhazé, Amine Rezrazi« Résultat d'un entraînement en conscience phonologique chez des élèves en difficultés de lecture au cycle 3 », Enfance 2014/2 $\left(\mathrm{N}^{\circ} 2\right)$, p. 119-134.

DOI $10.4074 / S 0013754514002018$

Distribution électronique Cairn.info pour NecPlus.

(C) NecPlus. Tous droits réservés pour tous pays.

La reproduction ou représentation de cet article, notamment par photocopie, n'est autorisée que dans les limites des conditions générales d'utilisation du site ou, le cas échéant, des conditions générales de la licence souscrite par votre établissement. Toute autre reproduction ou représentation, en tout ou partie, sous quelque forme et de quelque manière que ce soit, est interdite sauf accord préalable et écrit de l'éditeur, en dehors des cas prévus par la législation en vigueur en France. Il est précisé que son stockage dans une base de données est également interdit. 


\title{
Résultat d'un entraînement en conscience phonologique chez des élèves en difficultés de lecture au cycle 3
}

\author{
Sophie BRIQUET-DUHAZÉ* \\ et Amine REZRAZI**
}

\begin{abstract}
RÉSUMÉ
L'objet de cet article est de rapporter les résultats à mi-parcours d'un entraînement de trois ans portant sur le rôle de la conscience phonologique dans la maîtrise de la lecture chez des élèves de cycle 3 en difficulté. La conscience phonologique est la connaissance consciente et explicite du fait que les mots du langage oral sont composés d'unités plus petites (syllabes, rimes, phonèmes). Elle est un bon prédicteur en maternelle d'un apprentissage réussi de la lecture. Il s'agit ici de le transposer en remédiateur des difficultés en fin de scolarité élémentaire. Notre échantillon est composé de 300 élèves répartis en un groupe témoin et un groupe expérimental. Une analyse statistique des résultats intermédiaires met en évidence l'effet de l'entraînement après un an et demi.

MOTS-CLÉS : DIFFICULTÉS EN LECTURE, CONSCIENCE PHONOLOGIQUE, ÉLÈVES DE CYCLE 3, ÉDUCATION PRIORITAIRE.
\end{abstract}

\section{ABSTRACT}

\section{Result of phonological awareness training about pupils in difficulty aged 8,9 , and 10 years}

The purpose of this article is to report the mid-term results of a 3-year training on the role of phonological awareness in reading literacy among pupils with reading difficulty aged 8,9 and 10 years. Phonological awareness is defined as the conscious knowledge that the words of the oral language consist of smaller units (syllables, rhymes, phonemes). It is a good predictor for a successful mastery of reading. The training reported here aimed at evaluating whether phenomenological awareness can be used to remediate to difficulties at the end of the elementary cycle Our sample consists of 300 pupils distributed in control group and experimental group. A statistical analysis of the intermediate results highlights the effect of training after one and a half year.

KEY-WORDS: READING DIFFICULTIES, PHONOLOGICAL AWARENESS, PUPILS OF CYCLE 3, PRIORITY EDUCATION.

*Laboratoire CIVIIC, ESPE de l'Académie de Rouen. 2 rue du Tronquet, 76821 Mont-SaintAignan Cedex. Email: sophie.briquet@univ-rouen.fr

**laboratoire PsyNca, Université de R, 76821 Mont-Saint-Aignan Cedex. Email : amine.rezrazi@univ-rouen.fr 


\section{INTRODUCTION}

La conscience phonologique est une compétence métalinguistique qui consiste à opérer des tâches délibérées sur les mots à l'oral : reconnaissances des syllabes, rimes et phonèmes ainsi que toutes les opérations sur ces unités (permuter, ôter, ajouter...). La recherche scientifique montre de manière consensuelle que l'enfant doit savoir analyser intentionnellement le langage oral afin de réussir à apprendre à lire. En ce sens, la conscience phonologique est reconnue comme étant un prédicteur de l'apprentissage de la lecture. Cependant, l'intérêt porté aux élèves en difficulté du cycle trois (CE2-CM1-CM2), dans ce domaine précis, n'est que très peu étudié ; les recherches s'étant presque exclusivement concentrées sur les enfants du préscolaire ou en cours d'apprentissage de la lecture. Nous présentons successivement notre problématique et nos hypothèses permettant de transposer un prédicteur en remédiateur, le cadre théorique de la conscience phonologique, notre recherche longitudinale auprès d'élèves du cycle $3(9,10,11$ ans) et les résultats après traitement statistique auprès d'un échantillon d'élèves les plus en difficulté. Ces résultats sont enfin discutés au regard de notre cadre théorique.

\section{Problématique et hypothèses}

Les recherches dans ce domaine sont nombreuses, internationales, et leurs résultats concourants (Bradley et Bryant, 1991). La découverte du principe alphabétique qui consiste à faire le lien entre les phonèmes contenus dans les mots à l'oral et les lettres (Morais, 1994) est également essentielle.

Notre problématique s'inscrit donc dans ce contexte de vérification, d'autant que le nombre d'élèves en difficultés de lecture à l'entrée en $\sigma^{\mathrm{e}}$ reste constant (environ $15 \%$ ) depuis plusieurs années. Ainsi, l'étude spécifique réalisée à la demande de l'Observatoire national de la lecture (ONL), suite aux résultats des évaluations nationales annuelles en français, montre que, parmi les élèves en grande difficulté de lecture, trois groupes peuvent être différenciés (en 1997, ils sont 14,9\% en difficulté de lecture à l'entrée en sixième) : 4,3\% des élèves ont des grosses lacunes dans tous les domaines de la lecture ; 7,8\% ont des difficultés principalement liées à leur lenteur dans l'exécution des tâches ; 2,8 \% ont des difficultés moindres (Baktavatsalou \& Pons, 1999). Notons que le premier groupe commet de nombreuses erreurs en phonologie, mais ces performances augmentent au fur et à mesure lorsque l'on observe le dernier groupe constitué par les élèves en moins grande difficulté. Les performances en lecture (notamment) des élèves de CM2 ont été comparées entre 1987 et 2007 et font apparaître que, si les résultats sont stables durant les dix premières années, une baisse significative des scores s'observe entre 1997 et 2007, chez les élèves les plus fragiles (Rocher, 2008). De même, en 2008, les évaluations en lecture dans le cadre de la Journée d'appel de préparation à la défense (JAPD) font apparaître que $9,8 \%$ des jeunes ont des acquis limités en lecture (peu d'automatisation dans 
l'identification de mots principalement) et 11,8\% sont en difficulté de lecture (De La Haye, Gombert, Rivière, \& Rocher, 2009).

Qu'en est-il des compétences métaphonologiques des élèves de cycle trois ? En quoi l'entraînement de la conscience phonologique peut-il permettre à certains élèves en grande difficulté de progresser ? En fin d'école primaire, la maîtrise de la conscience phonologique est peu, voire non évaluée, et il est nécessaire de mentionner que les enseignants de ce cycle ne se sentent pas investis par cette tâche qu'ils estiment relever du cycle des apprentissages fondamentaux. Notre hypothèse repose donc sur le fait que même si l'entraînement de la conscience phonologique au cycle 3 ne peut enrayer toutes les difficultés en lecture d'autant qu'elles sont multicausales, il est vraisemblable que cet entraînement, bien que pratiqué tardivement, puisse avoir un effet positif et réel sur les compétences en lecture de certains élèves.

\section{CADRE THÉORIQUE DE LA CONSCIENCE PHONOLOGIQUE}

Pour apprendre à lire, il est nécessaire que l'enfant comprenne le principe alphabétique (caractérisant toutes les langues) consistant à faire correspondre une suite de lettres d'un mot lu en une suite de sons qui lui correspond (Rieben \& Perfetti, 1989). L'ensemble des correspondances graphèmes/phonèmes et de toutes les règles de combinaisons qui s’y rapportent en français caractérisent le code alphabétique (Giasson, 2012). En France, c'est au cycle 2 (GS, CP, CE1) que l'élève va apprendre cette procédure appelée communément le décodage. Plus cette procédure est automatisée, plus l'élève peut porter ses efforts sur la compréhension du texte lu (Gentaz \& Dessus, 2004). Cependant, le système alphabétique français n'est pas transparent; les lettres ne correspondent pas aux syllabes orales mais à des unités plus petites, les phonèmes. Découvrir le rôle de la conscience phonologique et connaître des lettres pour effectuer l'association des phonèmes à leurs unités écrites, sont deux conditions nécessaires à la découverte du principe alphabétique.

Gombert (1990, p. 29) définit la capacité métaphonologique comme étant « la capacité d'identifier les composants phonologiques des unités linguistiques et de les manipuler de façon délibérée ». L’identification et la manipulation volontaire des unités du mot (syllabes, rimes, phonèmes) sont donc deux capacités différentes, mais dont la maîtrise est tout aussi importante. Rey et Sabater (2007, p. 23) précisent que « la notion de conscience phonologique a fait l'objet de recherches multiples et variées, la plupart d'entre elles tentant d'établir une relation de causalité ou de réciprocité entre son développement et l'apprentissage de la lecture. Elles ont notamment contribué à mettre en évidence que la lecture est une tâche linguistique et que son apprentissage nécessite le développement, chez l'enfant, d'une conscience explicite et d'une manipulation intentionnelle des structures linguistiques ».

Un premier niveau de connaissances, au début de la scolarisation, concerne la manipulation et la prononciation des mots sur un plan épilinguistique (conscience 
implicite). Sur le plan métalinguistique (conscience explicite et donc étape supérieure), l'enfant doit manipuler les unités du mot de façon délibérée. La syllabe est aussi l'unité la plus aisée à repérer, par sa durée notamment (plus longue que le phonème) et son rôle dans la parole (accentuation). Par contre, les parties la constituant (attaque-rime (noyau-coda)) sont plus difficiles à détecter (Desrochers, Kirby, Thompson, \& Fréchette, 2009).

Nombreux sont les travaux scientifiques montrant une corrélation entre le niveau de conscience phonologique et la réussite de l'apprentissage de la lecture. Ils concernent largement la langue anglaise (Tunmer, 1991 ; Bryant, 1993 ; Castles \& Coltheart, 2004), mais aussi d'autres langues comme l'italien, l'allemand, l'espagnol... En français, les travaux sont moins nombreux mais convergents (Lecocq, 1986 ; Morais, 1994 ; Ecalle, 2000). Notons que les résultats de ces études doivent beaucoup au public d'enfants dyslexiques, pour lequel il a été constaté des difficultés importantes concernant ces traitements phonologiques (Lecocq, 1991 ; Sprenger-Charolles \& Casalis, 1996 ; Sprenger-Charolles \& Colé, 2006).

Liberman (1973), Liberman, Shankweiler et al. (1974) demeurent les auteurs de référence quant à l'identification des syllabes et des phonèmes. Il est demandé à des enfants, âgés de 4 à 6 ans, de taper sur la table le nombre de syllabes contenu dans un mot. Le nombre d'essais-erreurs nécessaire pour réaliser la tâche est calculé pour chaque tranche d'âge. Si le dénombrement de syllabes est bien réussi par tous, seuls les enfants de 6 ans parviennent à compter les phonèmes. Leur conclusion, comme celle d'autres auteurs (Vellutino \& Scalon, 1987 ; Tummer, 1989), est que ce développement serait plus tardif.

Les premiers travaux sur l'identification des rimes datent des années 1980 (Lenel \& Cantor, 1981) et concernent les enfants entre 3 et 7 ans. Les auteurs demandent à ces derniers de trouver parmi deux mots celui qui rime avec un autre également proposé. Le pourcentage de réussite est de plus de $60 \%$ à 3 ans pour voisiner les $90 \%$ vers 7 ans. Bradley et Bryant (1985) proposent à des enfants âgés de 4 à 5 ans de trouver un intrus parmi trois à quatre mots présentés oralement ou sous la forme d'un dessin. L'intrus est un mot qui ne rime pas, et l'entraînement consiste à manipuler des rimes. Le groupe expérimental a obtenu des résultats supérieurs au groupe contrôle d'environ une année scolaire.

Les épreuves de conscience phonologique appartiennent à trois catégories (Demont, Gaux, \& Gombert, 2006) :

- exercices de classification d'items ;

- exercices de segmentation et dénombrement (compter les syllabes d'un mot par exemple);

- exercices de manipulation (transformation d'un mot en ôtant un phonème ; élision phonémique, par exemple).

Cependant, la nature des unités les plus déterminantes à travailler (syllabes, rimes ou phonèmes) fait encore débat, comme le souligne Bara, Gentaz, \& Colé (2004). À cet égard, la conscience rimique demeure, comme pour Goswami (1993), 
d'une très grande importance, quand, pour d'autres auteurs, seule la conscience phonémique permet de prédire la réussite de l'apprentissage de la lecture (Ehri et al., 2001 ; Sprenger-Charolles \& Colé, 2006). Cette dernière voie semble être celle retenant le plus d'adhésions scientifiques.

L'entraînement de ces compétences métaphonologiques a un effet significatif sur la réussite en lecture, en augmentant particulièrement les compétences en décodage, en orthographe et en écriture (Lecocq, 1991). Les compétences en compréhension s'améliorent également du fait que celles-ci dépendent, en partie, du décodage rapide et juste des mots (Desrochers, Kirby, Thompson, \& Fréchette, 2009).

Dans les pays anglo-saxons, la période 2000-2010 favorise les méta-analyses. Le National Reading Panel (NRP) est chargé en 1997, de dresser un bilan de la recherche sur l'enseignement de la lecture, plus particulièrement les méthodes qui seraient les plus efficaces pour une application en classe. Les quatorze membres du NRP se sont appuyés sur les conclusions du Rapport du National Research Council (NRC) de Snow et al. 100000 travaux issus de la recherche ont été retenus ainsi que des auditions de 125 personnes (enseignants, parents, élèves. . .). Parmi les champs de recherche retenus, figurent :

- la méthode alphabétique : l'enseignement de la conscience phonologique et l'entraînement au décodage (correspondances $G / P$ ) ;

- la fluence (automatisation du processus de décodage);

- la compréhension (vocabulaire, texte);

- les logiciels d'apprentissage de la lecture.

Le NRP a, à l'analyse du corpus, relevé l'impact de l'apprentissage phonologique, de l'enseignement des correspondances lettres-sons, du lexique, des stratégies de compréhension, de la formation des enseignants en lecture et de l'impact de la lecture guidée à haute voix sur la fluence et la compréhension.

Concernant l'enseignement de la conscience phonologique, le NRP insiste sur la manipulation des phonèmes dans les syllabes et les mots et rappelle également le rôle prédicteur de la conscience phonologique et de la connaissance des lettres sur le niveau futur en lecture, d'autant que la phonologie est indispensable au décodage.

En 2008, un rapport du Comité national d'alphabétisation précoce (NELP : National Early Literacy Panel) est publié. Ce comité réalise une méta-analyse débutée en 2002, consistant à mettre au jour parmi les 8000 articles publiés dans des revues à comité de lecture, les recherches montrant une corrélation entre alphabétisation précoce et résultats ultérieurs en littéracie. Ce comité, convoqué par le National Institute for Literacy, était chargé de déterminer les pratiques pédagogiques permettant le développement de compétences en littéracie précoce. La méthodologie a consisté à sélectionner une partie de ces recherches au regard des critères scientifiques (500 retenues) et de les coder afin de permettre leur comparaison et l'analyse statistique. 
Les principaux résultats portent sur ce qui est appelé en américain « la littéracie conventionnelle » à savoir le décodage, la fluence, la compréhension, l'orthographe et l'écriture. Les six variables les plus fortement corrélées à la réussite future en lecture-écriture dans ces domaines sont : la conscience phonologique, la dénomination rapide de lettres ou de chiffres, la dénomination rapide d'objets et de couleurs, la mémoire de travail phonologique, la connaissance de l'alphabet (nom et son des lettres), l'écriture de lettres isolées ou l'écriture de son prénom en dictée. Côté interventions pédagogiques les plus efficaces, celles consistant à l'apprentissage systématique des correspondances lettres-sons sont les seules ayant un impact significatif (modéré à fort) sur le décodage, la fluence, la compréhension, l'orthographe et l'écriture. Le comité précise qu'elles sont plus efficaces lorsqu'elles sont dispensées par l'enseignant, en petits groupes à l'occasion de tâches simples de lecture de mots impliquant l'apprentissage de l'alphabet et la manipulation phonémique.

Comme l'atteste Desrochers, Kirby, Thompson et Fréchette (2009), le niveau de conscience phonologique est plus faible chez les enfants issus de milieux défavorisés, car moins exposés à la langue orale et écrite dans leur environnement. Des programmes d'entraînement, favorisant le développement de la conscience phonologique et l'apprentissage des lettres à l'école, devraient permettre de réduire les risques d'échec en lecture, ce qui est montré par beaucoup de travaux (Bus \& Van Ijzendoorn, 1999). Cela ne résout pas toutes les difficultés liées à un environnement plus modeste mais les réduit significativement. Le décodage phonologique est ainsi enclenché (procédure analytique) et surtout les élèves ont confiance en eux. Il manquera la prononciation rapide et entière des mots et l'accès à leur sens (procédure lexicale), sachant qu'un mot ne peut être compris à l'écrit que si son sens est déjà connu à l'oral.

\section{MÉTHODOLOGIE}

\section{Sujets (ou participants)}

Notre échantillon est composé de 300 élèves de CE2 répartis au sein de deux groupes (expérimental : 235 élèves et témoin : 65 élèves). Ces élèves présentent des résultats faibles en lecture aux évaluations nationales. Le groupe expérimental est subdivisé en deux, l'un suit un entraînement en conscience phonologique et de la connaissance du nom des lettres (110 élèves) tandis que l'autre suit uniquement un entraînement de la conscience phonologique (125 élèves). Quatre écoles sur les cinq constituant notre groupe expérimental appartiennent au réseau Éclair ${ }^{1}$.

\section{Matériel}

L'évaluation initiale en début de CE2 a été réalisée en connaissance du nom des lettres et conscience phonologique complétée par une épreuve en lecture oralisée

1 Éclair : Écoles, collèges et lycées pour l’ambition, l’innovation et la réussite. 
de phrases, de mots réguliers, irréguliers et de pseudo-mots. La rapidité de la réponse et la justesse ont été les deux critères retenus comme lors de la phase 0 (l'hésitation, la bonne réponse en deuxième reprise. . n’ont pas été considérés par exemple).

En CM1, l'évaluation intermédiaire (décembre 2012, en annexe 1) nous a permis de tester à nouveau la moitié des élèves en difficulté $(\mathrm{N}=42)$; l'évaluation terminale aura lieu en juin 2014 au CM2.

\section{Procédure}

Suite à l'évaluation initiale en CE2, les enseignants en charge de ces classes ont commencé l'entraînement selon un protocole décrit dans un fichier conçu par d'autres enseignants et un des chercheurs. Pour ce faire, les professeurs des écoles ont suivi une animation pédagogique sur ce thème et des réunions périodiques sont organisées. L'entraînement commence par la notion de syllabes (dénombrement-fusion-repérage de syllabes identiques - ôter une syllabe) puis la notion de rimes est approfondie (mots qui riment avec un autre - qui ne riment pas). L'entraînement durant l'année de CE2 se termine par l'étude du premier phonème [a] (reconnaître-localisersubstituer-inverser-ôter-ajouter). L'ensemble propose une trentaine de jeux réalisés par l'ensemble des élèves (sans distinction par rapport aux résultats des évaluations individuelles) dans un cadre ludique et en fonction du rythme propre à chaque classe. Seules sont imposées la fréquence et la durée : deux fois vingt minutes deux fois par semaine. L'apprentissage a lieu en boucle c'est-à-dire que les jeux étudiés sont partiellement repris lors des séances suivantes pour une meilleure imprégnation et un renforcement de l'automatisation.

Après l'évaluation intermédiaire en CM1 les enseignants ont repris quelques jeux du premier fichier étudié en CE2 et ont commencé l'étude des phonèmes dans l'ordre de fréquence (Reprise du phonème [a] en 1 ; phonème 2 : [r] ; phonème $3:[1]$; phonème $4:[\mathrm{e}]$; phonème $5:[\mathrm{s}])$. L'ordre des phonèmes et leur choix sont dictés par notre analyse de leur fréquence dans les textes en français. En effet, notre hypothèse repose sur le fait que l'étude aléatoire des phonèmes, l'année de l'apprentissage de la lecture, peut poser problème aux élèves de $\mathrm{CP}$ susceptibles d'être en difficulté. Un ancien tableau provenant d'un ouvrage de 1970 de Genouvrier et Peytard ${ }^{2}$, est riche d'enseignement. Il s'agit du tableau de fréquence des phonèmes à l'oral, les auteurs précisant que pour l'écrit, il n'y a pratiquement aucune différence sauf pour le [ə]. Lors de l'apprentissage de la lecture, en abordant les phonèmes dans l'ordre de fréquence, cela permet d'automatiser $34,1 \%$ du décodage lorsque les 5 premiers phonèmes sont étudiés et 58,9\% lorsque les 10 premiers sur les 35 que comptent la langue française, sont appris.

2 Genouvrier E. et Peytard J. (1970). Linguistique et enseignement du français. Paris : Larousse, p. 42. 


\section{RÉSULTATS}

Des analyses préliminaires n'ont révélé aucun effet notable de la condition expérimentale sur les performances linguistiques des élèves à la première évaluation en CE2. En effet, l'analyse de comparaison des moyennes de l'indice global de compétence entre les trois groupes (deux expérimentaux et un groupe contrôle) est non significative $\left[\mathrm{F}_{(2,39)}=2,34 ; p=0,11 ; \eta^{2}=0,11\right]$. De même, les analyses de variances des différentes compétences linguistiques ont monté que les performances des trois groupes sont relativement proches pour les quatre notions linguistiques au CE2 : notion de mots $\left[\mathrm{F}_{(2,39)}=2,11 ; p=0,14\right.$; $\left.\eta^{2}=0,09\right]$, notion de rimes $\left.\mathrm{F}_{(2,39)}=0,73 ; p=0,49 ; \eta^{2}=0,04\right]$, notion de syllabes $\left[\mathrm{F}_{(2,39)}=2,2 ; p=0,12 ; ; \eta^{2}=0,10\right]$ et notion de phonèmes $\left[\mathrm{F}_{(2,39)}=\right.$ 1,$\left.19 ; p=0,31 ; \eta^{2}=0,06\right]$.

\section{Compétence linguistique globale}

On a relevé, pour chaque élève, le score de réussite aux huit items proposés. Les données ont été soumises à une analyse de variance à 2 facteurs : groupe expérimental (témoin, conscience phonologique (ECP), conscience phonologique et nom des lettres (ECPL) x périodes d'évaluation (CE2, CM1) avec mesures répétées sur ce dernier facteur. Les résultats montrent un effet période significatif $\left[\mathrm{F}_{(1,39)}=86,47 ; p<0,001 ; \eta^{2}=0,70\right]$, tous les élèves améliorent leur score entre le CE2 $(\mathrm{m}=2,30$; ET $=0,65)$ et le CM1 $(\mathrm{m}=4,29$; $\mathrm{ET}=1,31)$. De même, l'effet du facteur groupe est aussi significatif $\mathrm{F}_{(2,39)}=$ 6,$\left.05 ; p<0,01 ; \eta^{2}=0,24\right]$, les performances des groupes qui ont suivi les protocoles d'entraînements (ECP : $\mathrm{m}=3,54$; $\mathrm{ET}=$; ECPL : $\mathrm{m}=3,77$; $\mathrm{ET}=0,95)$ sont supérieures à celles du groupe témoin $(\mathrm{m}=2,67 ; \mathrm{ET}=1,02)$ ( $c f$. fig. 1). Les tests post-hoc confirment l'effet bénéfique de l'entraînement phonologique sur la progression de la performance linguistique des élèves. En effet, le test de Bonferroni montre une différence significative entre le groupe témoin, aussi bien avec le groupe ECP $(\phi<0,05)$ qu'avec le groupe ECPL $(p$ $<0,01)$, tandis que la comparaison entre les groupes ECP et ECPL est non significative $(p>0,1)$. Conformément à notre hypothèse, on constate un effet d'interaction significatif entre le facteur période et le facteur groupe $\left[\mathrm{F}_{(2,39)}=\right.$ 5,$\left.41 ; p<0,01 ; \eta^{2}=0,22\right]$.

Afin d'évaluer l'effet d'interaction de l'entraînement phonologique sur la progression linguistique des élèves, nous avons séparé les élèves en deux catégories : les élèves qui ont amélioré leur score de compétences linguistiques entre le CE2 et le CM1 (progression) et les élèves qui ont obtenu le même score ou moins en CM1 qu'en CE2 (stagnation/régression). Le tableau 1 indique la répartition des élèves en fonction de ces deux catégories.

L'évaluation en CM1 indique une réelle progression chez les élèves qui ont participé aux protocoles d'entraînement phonologique. Le taux d'amélioration des performances linguistiques est supérieur chez les élèves du groupe ECP que chez les élèves du groupe témoin $(100 \%$ vs $38 \%)\left[p_{\text {Fisher exact }}<0,01\right]$. On constate 


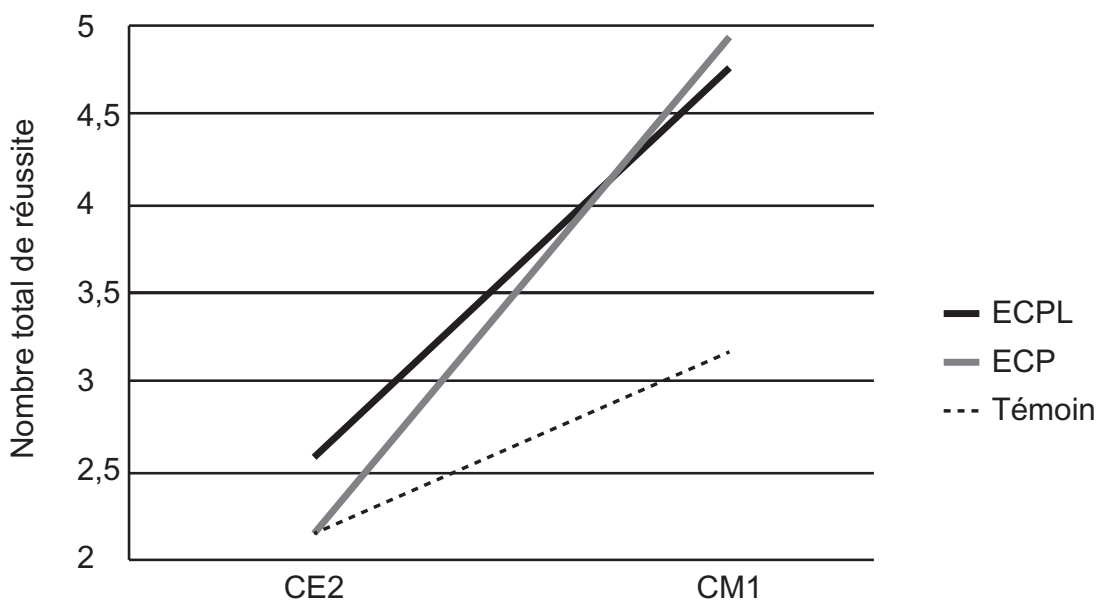

Figure 1.

Nombre d'items réussis selon la condition expérimentale

\section{Tableau 1.}

Le taux de progression entre les deux évaluations selon la condition expérimentale

\begin{tabular}{lcccc}
\hline & progression & stagnation/régression & $\begin{array}{c}\text { Fisher exact } \\
\text { Témoin/ECP }\end{array}$ & $\begin{array}{c}\text { Fisher exact } \\
\text { Témoin/ECPL }\end{array}$ \\
\hline $\mathrm{ECP}(\mathrm{n}=12)$ & $12(100 \%)$ & $0(0 \%)$ & $\mathrm{p}<0,01$ & \\
témoin $(\mathrm{n}=13)$ & $5(38 \%)$ & $7(62 \%)$ & & $\mathrm{p}<0,01$ \\
$\mathrm{ECPL}(\mathrm{n}=17)$ & $15(88 \%)$ & $2(12 \%)$ & & \\
\hline
\end{tabular}

que la différence est aussi significative entre le groupe ECPL (88\%) et le groupe témoin $\left[p_{\text {Fisher exact }}<0,01\right]$.

Les notions linguistiques

Le tableau 2 présente les résultats de l'analyse de variance à mesure répétée sur les scores des élèves aux quatre notions linguistiques évaluées en CE2 et en CM1 : mots, rimes, syllabes et phonèmes.

On constate des effets significatifs du facteur groupe sur les notions de mots et de syllabes, ainsi que des effets significatifs d'interaction. Les résultats démontrent que la progression entre les deux niveaux varie en fonction du groupe expérimental. Chez les élèves du groupe témoin le taux moyen de réussite a diminué lors de la deuxième évaluation (moins $58 \%$ pour la notion de mots et moins $20 \%$ pour la notion de syllabes). Alors que les élèves qui ont bénéficié de l'entraînement phonologique augmentent notablement leurs scores en CM1 pour les deux compétences linguistiques. 


\section{Tableau 2.}

Moyennes des notions linguistiques

en fonction de la condition expérimentale et du niveau scolaire

\begin{tabular}{|c|c|c|c|c|c|}
\hline & $\begin{array}{c}\text { CE2 } \\
\text { Moy. (ET) }\end{array}$ & $\begin{array}{c}\text { CM1 } \\
\text { Moy. (ET) }\end{array}$ & $\begin{array}{l}\text { Effet période } \\
\mathbf{F}_{(1.39)} / \eta^{2}\end{array}$ & $\begin{array}{l}\text { Effet groupe } \\
\mathbf{F}_{(2.39)} / \eta^{2}\end{array}$ & $\begin{array}{c}\text { Effet } \\
\text { interaction } \\
\mathbf{F}_{(2.39)} / \eta^{2}\end{array}$ \\
\hline \multicolumn{6}{|c|}{ Notion de mots (1 item) } \\
\hline Témoin & $0,17(0,39)$ & $0,08(0,29)$ & $\begin{array}{l}F=11,84^{* *} \\
\eta^{2}=0,23\end{array}$ & $\begin{array}{l}F=4,92^{*} \\
\eta^{2}=0,20\end{array}$ & $\begin{array}{l}F=4,65^{*} \\
\eta^{2}=0,19\end{array}$ \\
\hline ECP & $0,23(0,44)$ & $0,69(0,48)$ & & & \\
\hline ECPL & $0(0)$ & $0,53(0,51)$ & & & \\
\hline \multicolumn{6}{|c|}{ Notion de rimes (2 items) } \\
\hline Témoin & $0(0)$ & $0,83(0,84)$ & $\begin{array}{l}F=79,11 * * * \\
\eta^{2}=0,67\end{array}$ & $\begin{array}{l}F=1,285 \\
\eta^{2}=0,06\end{array}$ & $\begin{array}{l}F=1,46 \\
\eta^{2}=0,07\end{array}$ \\
\hline ЕСР & $0(0)$ & $1,31(0,63)$ & & & \\
\hline ECPL & $0,06(0,154)$ & $1,01(0,71)$ & & & \\
\hline \multicolumn{6}{|c|}{ Notion de syllabes (3 items) } \\
\hline Témoin & $1,67(0,65)$ & $1,33(0,49)$ & $\begin{array}{l}F=1,13 \\
\eta^{2}=0,03\end{array}$ & $\begin{array}{l}F=10,91^{* * *} \\
\eta^{2}=0,36\end{array}$ & $\begin{array}{l}F=3,54^{*} \\
\eta^{2}=0,16\end{array}$ \\
\hline ECP & $1,46(0,52)$ & $1,77(0,59)$ & & & \\
\hline ECPL & $1,69(0,56)$ & $2,29(0,47)$ & & & \\
\hline \multicolumn{6}{|c|}{ Notion de phonème (2 items) } \\
\hline Témoin & $0,33(0,65)$ & $0,92(0,79)$ & $\begin{array}{l}F=15,97 * * * \\
\eta^{2}=0,29\end{array}$ & $\begin{array}{l}F=1,06 \\
\eta^{2}=0,05\end{array}$ & $\begin{array}{l}F=0,59 \\
\eta^{2}=0,03\end{array}$ \\
\hline ЕСР & $0,46(0,52)$ & $1,15(0,69)$ & & & \\
\hline ECPL & $0,65(0,55)$ & $1,01(0,35)$ & & & \\
\hline
\end{tabular}

${ }^{*} p<0,05 ;{ }^{* *} p<0,01 ;{ }^{* * *} p<0,001$

On note des effets significatifs du facteur période d'évaluation sur les performances des notions de rimes et phonèmes. Tous les élèves ont augmenté sensiblement leurs scores entre le CE2 et le CM1. Par contre, l'influence du facteur groupes et les effets d'interactions sont négligeables.

Pour chacun des trois groupes, nous avons comptabilisé le pourcentage des élèves qui ont amélioré leur performance de mots et de syllabes entre le CE2 et CM1 (cf. fig2)

On constate que les élèves maîtrisent davantage les mots et les syllabes dans la condition expérimentale que dans la condition contrôle $\left[\phi_{\text {Fisher exact }}<0,05\right]$. On remarque qu'un élève sur 12 du groupe témoin progresse autant dans le mot que dans la syllabe ; par contre, la progression est beaucoup plus importante chez les élèves des groupes expérimentaux : ECP ( 7 élèves pour le mot et 6 élèves pour la syllabe) ; ECPL (7 élèves pour le mot et 7 élèves pour la syllabe). Par ailleurs, on note que l'entraînement phonologique permet non seulement de maîtriser le langage écrit, mais il permet surtout de consolider les acquis du langage. En effet, aucun élève du groupe ECPL n'a subi une perte de performance sur la notion de syllabe ; de même, chez le groupe ECP, la diminution n'a été constatée que chez un seul élève. En revanche, cette régression de la notion de syllabes est beaucoup 


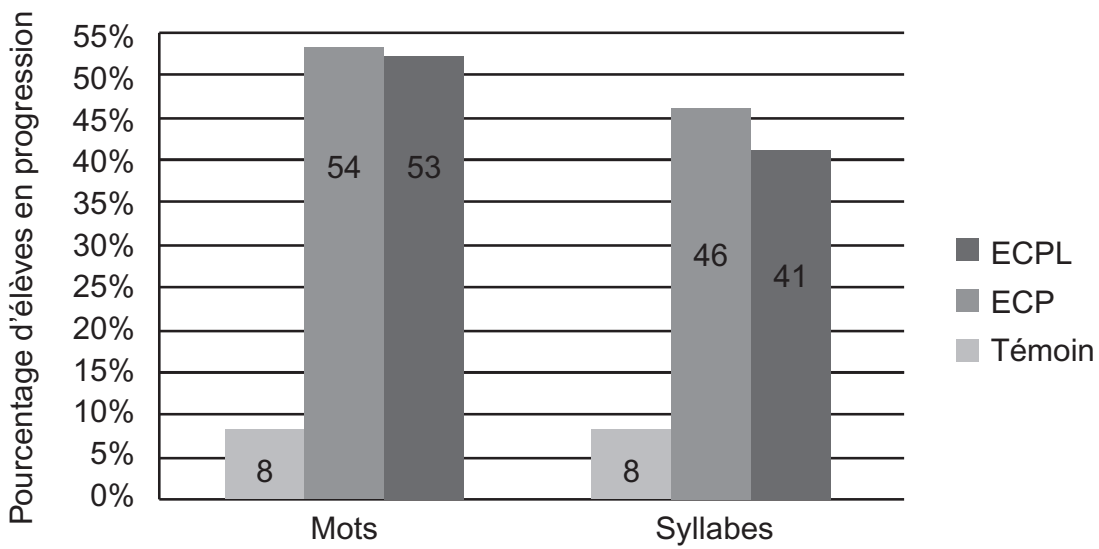

\section{Figure 2.}

Pourcentage des élèves qui ont progressé dans les notions de mots et de syllabes entre le CE2 et le CM1 en fonction de la condition expérimentale

plus importante chez le groupe témoin puisque $50 \%$ des élèves subissent une perte de performance syllabique entre le CE2 et CM1.

\section{Discussion}

Les élèves des groupes expérimentaux (ECP et ECPL) progressent significativement entre le CE2 et le CM1. La décomposition des items nous permet, pour chacun des trois groupes, de repérer ceux pour lesquels les progrès sont significatifs. Les groupes expérimentaux améliorent significativement les items 6 (suppression de la dernière syllabe), 7 (discrimination d'un phonème) et 1 (notion de mot).

À cela, il faut ajouter que l'item 5 (fusion de syllabes) est aggravé pour le groupe témoin et amélioré pour le groupe ECPL. La fusion de syllabes est importante à automatiser car elle sert lors du décodage à former le mot, savoir où couper les mots de manière à donner du sens à ce qui est lu. Un décodage mécaniste n'apporte pas de sens (ONL, 1998). La deuxième fonction de la fusion de syllabes est de permettre l'encodage d'un mot, d'une phrase, c'est-à-dire la capacité de l'élève à pouvoir mentalement découper un mot afin d'en extraire la première syllabe, puis, appliquer la conversion phonème/graphème avant de retrouver la seconde syllabe et d'appliquer le même principe. La fusion est une opération favorisant ces allers-retours. La fusion de syllabes dans le désordre permet à l'enfant de rechercher du sens plutôt que d'appliquer une fusion uniquement liée à l'application du principe de l'ordre. Nous pouvons émettre l'hypothèse que l'apprentissage du nom des lettres en complément de la conscience phonologique favoriserait la fusion de syllabes dans l'ordre et le désordre car les écarts de performances, en CM1, sont importants chez les élèves en difficultés issus des trois groupes. Ainsi, nous remarquons qu'au cycle 3, la syllabe reste l'unité la plus facile à maittriser ; ces résultats concordent avec ceux 
de l'effet prédicteur (Desrochers et al., 2009). Cependant, beaucoup de chercheurs s'accordent sur le fait que le développement des phonèmes serait plus tardif (Vellutino \& Scalon, 1987 ; Tummer, 1989). Notre recherche montre que si cet entraînement ne perdure pas au cycle 3, la notion de phonème n'est pas plus acquise qu'avant l'apprentissage de la lecture et la maîtrise de la notion de syllabe passablement acquise antérieurement peut se dégrader sans entraînement. L'ensemble de ces résultats et plus particulièrement les performances des items 7 et 8 rejoignent les travaux de Desrochers, Kirby, Thompson et Fréchette (2009) montrant que le niveau de conscience phonologique est plus faible chez les enfants issus de milieux défavorisés. Si un entraînement favorisant le développement de la conscience phonologique et la connaissance des lettres peut permettre de réduire les risques d'échec en lecture (Bus \& Van Ijzendoorn, 1999), nos propres résultats auprès d'élèves scolarisés en milieu difficile montrent qu'en aval cet entraînement n'est pas neutre lorsqu'il a lieu de manière régulière et sur le long terme.

\section{Conclusion}

Notre recherche sur l'entraînement de la conscience phonologique et la connaissance du nom des lettres s'achèvera en juin 2014. Le traitement statistique opéré nous permettra de mettre au jour certaines corrélations et de comparer les groupes en fonction de l'évaluation initiale (CE2, novembre décembre 2011), intermédiaire (CM1, décembre 2012-janvier 2013) et terminale (CM2, juin 2014). Cela permettra surtout d'évaluer l'impact de cette remédiation sur les résultats en lecture ; ce qui est l'objectif principal de cette étude. Nous disposons pour cela des évaluations en conscience phonologique, en connaissance du nom des lettres, en lecture à voix haute de phrases, de mots réguliers, irréguliers et de pseudo-mots.

Si nos premiers résultats montrent une amélioration significative des élèves les plus en difficulté appartenant aux groupes expérimentaux après un an et demi d'entraînement (entre le CE2 et le CM1), il nous faudra, bien sûr, attendre la fin de l'entraînement afin de mesurer les différences ou non entre le niveau initial et terminal chez l'ensemble des élèves des trois groupes. Au-delà, ces résultats contredisent la non-efficacité d'un entraînement de la conscience phonologique chez des élèves plus âgés. Cette ultime analyse nous fournira pour les trois groupes, le taux de progression en décodage notamment.

\section{BIBLIOGRAPHIE}

Baktavatsalou, R., \& Pons, C. (1999). Étude spécifique sur les élèves en difficulté en lecture à l'entrée en sixième, Note d'information, 48, Paris : MEN, DEPP.

Bara, F., Gentaz, E., \& Colé, P. (2004). Les effets des entraînements phonologiques et multisensoriels destinés à favoriser l'apprentissage de la lecture chez les jeunes enfants, Enfance, 56(4), 387-403.

Bradley, L., \& Bryant, P. (1985). Rhyme and reason in reading and spelling. Ann Arbor, MI: The University of Michigan Press. 
Bradley, L., \& Bryant, P. (1991). Phonological skills before and after learning to read. In S., Brady, D. Shankweiler (éds.), Phonological processes in literacy: a tribute to Isabelle Y. Liberman (p p. 1-34), Hillsdale, New Jersey: Laurence Erlbaum Associates.

Bryant, P. (1993). Conscience phonologique et apprentissage de la lecture. in J. P. Jaffré, L. Sprenger-Charolles, Fayol M. (éds.), Lecture-écriture : acquisition (p p. 176192), Paris: Nathan.

Bus, A. G. \& Van Ijzendoorn, M. H. (1999). Phonological awareness and early reading: a meta-analysis of experimental training studies, Journal of Educational Psychology, 91(3), 403-414.

Castles, A., \& Coltheart, M. (2004). Is there a causal link from phonological awareness to success in learning to read? Cognition, 91, 77-111.

De La Haye, F., Gomber, t J. E., \& Rivière, J. P. (2009). Les évaluations en lecture dans le cadre de la journée d'appel de préparation à la défense (année 2008), Note d'information, 09-19. Paris: MEN-DEPP, juillet.

Demont, E., Gaux, C., \& Gombert J. E. (2006). Bilan métalinguistique. in F. Estienne, Piérart B. (dir.), Les bilans de langage et de voix : fondements théoriques et pratiques (pp. 105-122), Paris: Masson.

Desrochers, A., Kirby, J. R., Thompson, G. L., \& Fréchette, S. (2009). Le rôle de la conscience phonologique dans l'apprentissage de la lecture, Revue du NouvelOntario, 34, 59-82.

Ecalle, J. (2000). Prédiction de réussite scolaire en lecture-écriture au cycle 2, Revue Européenne de psychologie appliquée, 50, 81-85.

Ehri, L. C., Nunes, S. R., Willows, D. M., Schster, D. M., Yaghoub-Zadeh, Z., \& Shanahan, T. (2001). Phonemic awareness instruction helps children learn to read: evidence from the National Reading Panel's meta-analysis, Reading Research Quarterly, 36, 250-287.

Gentaz, E., Dessus, P. (éds.) (2004). Comprendre les apprentissages. Sciences cognitives et éducation. Paris : Dunod.

Giasson, J. (2012). La lecture. Apprentissage et difficultés. Bruxelles : De Boeck.

Gombert, J. E. (1990). Le développement métalinguistique. Paris : PUF.

Goswami, U. (1993). Toward an interactive analogy model of reading development: decoding wowel graphemes in beginning reading, Journal of Experimental Child Psychology, 56, 217-240.

Lecocq, P. (1986). Sensibilité à la similarité phonétique chez les enfants dyslexiques et les bons lecteurs, L'Année psychologique, 86, 201-221.

Lecocq, P. (1991). Apprentissage de la lecture et dyslexie. Liège : Mardaga.

Lenel, J. C., \& Cantor, J. H. (1981). Rhyme recognition and phonemic perception in young children, Journal of Psycholinguistic Research, 10, 57-67.

Liberman, I. Y. (1973). Segmentation of the spoken word and reading acquisition, Bulletin of the Orton Society, 23, 65-77.

Liberman, I. Y., Shankweiler, D., Fisher, F. W., \& Carter, B. (1974). Explicit syllable and phoneme segmentation in the young child, Journal of Experimental Child Psychology, 18, 201-212.

Morais, J. (1994). L'art de lire. Paris : Odile Jacob.

National Early Literacy Panel (2008). Developing early literacy. Washington: National Institute for Literacy, $234 \mathrm{p}$.

National Reading Panel (2000). Teaching Children to read: Evidence-Based Assessment of the Scientific Research Literature on Reading and its implications for Reading Instruction.

enfance $n^{\circ} 2 / 2014$ 
A report of Subgroup. Washington, DC: National Institute of Child Health and Human Development, 449 p.

Rey, V., \& Sabater, C. (2007). Conscience phonologique, conscience morphologique et apprentissage de la lecture : état de la question, Glossa, 100, p. 22-35.

Rieben, L., \& Perfetti, L. (1989). L'apprenti lecteur. Neuchâtel/Paris : Delachaux et Niestlé.

Rocher, T. (2008). Lire, écrire, compter : les performances des élèves à vingt ans d'intervalle 1987-2007, Note d'information, 38, Paris : MEN, DEPP.

Sprenger-Charolles, L., \& Casalis, S. (1996). Lecture et écriture : acquisition et troubles du développement. Paris : PUF.

Sprenger-Charolles, L., \& Colé, P. (2006). Lecture et dyslexie. Approche cognitive. Paris: Dunod.

Tunmer, W. E. (1991). Phonological awareness and literacy acquisition. in L. Rieben, Perfetti C.A. (eds.). Learning to read (pp. 105-119), New Jersey: Lawrence Erlbaum Associates.

Tunmer, W. E. (1989). Conscience phonologique et acquisition de la langue écrite. in L. Rieben, C.A. Perfetti. L'apprenti-lecteur : recherches empiriques et implications pédagogiques (pp. 197-215), Neuchâtel/Paris: Delachaux et Niestlé.

Vellutino, F. R., \& Scanlon, D. M. (1987). Phonological coding, phonological awareness and reading ability: evidence from a longitudinal and experimental study, Merrill-Palmer Quaterly, 33, 321-363.

\section{Annexe 1 : évaluation intermédiaire CM1}

\section{Notion de mot :}

Consigne : «Dis-moi combien il y a de mots dans les phrases que je vais dire à l'oral ?"

Tu lances une bal

Réponse :

Les enfants récitent leur leçon.

Elle se couche tard.

Réponse :

Réponse :

Épreuve 1

(Dénombrement de mots)

2. Notion de rimes :

Consigne : «Trouve 2 mots qui riment avec le mot gâteau »

(L'enseignant écrit les propositions orales de l'enfant)

Réponses :

Épreuve 2

(Association mot/rime)

Consigne : "Trouve un mot qui rime avec... »

Caillou

Couleur

Océan
Réponse :

Réponse :

Réponse : 
3. Notion de syllabe :

Consigne : «Combien de syllabes y a-t-il dans le mot. . ? »
Maison
Jardin
Médecin
Dessiner
Porte
Voiture
Réponse :
Réponse :
Réponse :
Réponse :
Réponse :
Réponse :

Épreuve 4

(Dénombrement de syllabes)

Consigne : "Je te donne plusieurs syllabes, dis-moi quel mot on obtient lorsqu'on les met ensemble?»

Len-de-main

Réponse :

De-man-der

Réponse :

Veau-nou

Seau-oi

Réponse :

Vé-té-ri

Réponse :

Réponse :

Consigne : " je te donne un mot composé de plusieurs syllabes, tu supprimes la dernière syllabe et tu me dis quel mot il reste. Voici un exemple : rideau è riz »
Garçon
Couleur
Demain
Certain
Leçon
Réponse :
Réponse :
Réponse :
Réponse :
Réponse :
Épreuve 6
(Suppression de syllabes)

4. Notion de phonèmes :

Consigne : je prononce quatre mots, l'un d'entre eux ne commence pas par le même son que les autres, dis-moi lequel. Voici un exemple : ramer, rond, cerise, rouge. C'est « cerise » car ce mot ne commence pas par le son (phonème) [r].

Soleil, sonner, sourire, cher.

Manger, salade, malade, madame

Visage, voisin, message, village
Réponse :

Réponse :

Réponse :

Épreuve 7

(Identification de phonèmes) 
Consigne : je prononce un mot ; enlève le dernier son et dis-moi le mot que l'on obtient. Voici deux exemples : carré, j'enlève «é », il reste car ; noir, j'enlève « $\mathbf{r} »$, il reste noi( $\mathbf{x})$.

Voilà

Sur

Profond

Matin
Réponse :

Réponse :

Réponse :

Réponse :

Épreuve 8

(Suppression de phonème) 